\title{
Multiple positive solutions for a class of quasi- linear elliptic equations involving concave-convex nonlinearities and Hardy terms
}

\section{Tsing-San Hsu}

Correspondence: tshsu@mail.cgu. edu.tw

Center for General Education, Chang Gung University, Kwei-San, Tao-Yuan 333, Taiwan ROC

\section{Abstract}

In this paper, we are concerned with the following quasilinear elliptic equation

$$
-\Delta_{p} u-\mu \frac{|u|^{p-2} u}{|x|^{p}}=\lambda f(x)|u|^{q-2} u+g(x)|u|^{p *-2} u \text { in } \Omega, \quad u=0 \text { on } \partial \Omega,
$$

where $\Omega \subset \mathbb{R}^{N}$ is a smooth domain with smooth boundary $\partial \Omega$ such that $0 \in \Omega, \Delta_{p} U$ $=\operatorname{div}\left(|\nabla u|^{p-2} \nabla u\right), 1<p<N, \mu<\bar{\mu}=\left(\frac{N-p}{p}\right)^{p}, \lambda>0,1<q<p$, sign-changing weight functions $f$ and $g$ are continuous functions on $\bar{\Omega}, \bar{\mu}=\left(\frac{N-p}{p}\right)^{p}$ is the best Hardy constant and $p^{*}=\frac{N p}{N-p}$ is the critical Sobolev exponent. By extracting the Palais-Smale sequence in the Nehari manifold, the multiplicity of positive solutions to this equation is verified.

Keywords: Multiple positive solutions, critical Sobolev exponent, concave-convex, Hardy terms, sign-changing weights

\section{Introduction and main results}

Let $\Omega$ be a smooth domain (not necessarily bounded) in $\mathbb{R}^{N}(N \geq 3)$ with smooth boundary $\partial \Omega$ such that $0 \in \Omega$. We will study the multiplicity of positive solutions for the following quasilinear elliptic equation

$$
\left\{\begin{array}{lr}
-\Delta_{p} u-\mu \frac{|u|^{p-2} u}{|x|^{p}}=\lambda f(x)|u|^{q-2} u+g(x)|u|^{p *-2} u, & \text { in } \Omega, \\
u=0, & \text { on } 2 \Omega,
\end{array}\right.
$$

where $\Delta_{p} u=\operatorname{div}\left(|\nabla u|^{p-2} \nabla u\right), 1<p<N, \mu<\bar{\mu}=\left(\frac{N-p}{p}\right)^{p}, \bar{\mu}$ is the best Hardy constant, $\lambda>0,1<q<p, p^{*}=\frac{N p}{N-p}$ is the critical Sobolev exponent and the weight functions $f, g: \bar{\Omega} \rightarrow \mathbb{R}$ are continuous, which change sign on $\Omega$.

Let $\mathcal{D}_{0}^{1, p}(\Omega)$ be the completion of $C_{0}^{\infty}(\Omega)$ with respect to the norm $\left(\int_{\Omega}|\nabla u|^{p} d x\right)^{1 / p}$. The energy functional of (1.1) is defined on $\mathcal{D}_{0}^{1, p}(\Omega)$ by

$$
J_{\lambda}(u)=\frac{1}{p} \int_{\Omega}\left(|\nabla u|^{p}-\mu \frac{|u|^{p}}{|x|^{p}}\right) \mathrm{d} x-\frac{\lambda}{q} \int_{\Omega} f|u|^{q} \mathrm{~d} x-\frac{1}{p^{*}} \int_{\Omega} g|u|^{p^{*}} \mathrm{~d} x
$$

Then $J_{\lambda} \in C^{1}\left(\mathcal{D}_{0}^{1, p}(\Omega), \mathbb{R}\right) \cdot u \in \mathcal{D}_{0}^{1, p}(\Omega) \backslash\{0\}$ is said to be a solution of (1.1) if $\left\langle J_{\lambda}^{\prime}(u), v\right\rangle=0$ for all $v \in \mathcal{D}_{0}^{1, p}(\Omega)$ and a solution of (1.1) is a critical point of $J_{\lambda}$.

(c) 2011 Hsu; licensee Springer. This is an Open Access article distributed under the terms of the Creative Commons Attribution License (http://creativecommons.org/licenses/by/2.0), which permits unrestricted use, distribution, and reproduction in any medium, provided the original work is properly cited. 
Problem (1.1) is related to the well-known Hardy inequality [1,2]:

$$
\int_{\Omega} \frac{|u|^{p}}{|x|^{p}} \mathrm{~d} x \leq \frac{1}{\bar{\mu}} \int_{\Omega}|\nabla u|^{p} \mathrm{~d} x, \quad \forall u \in C_{0}^{\infty}(\Omega) .
$$

By the Hardy inequality, $\mathcal{D}_{0}^{1, p}(\Omega)$ has the equivalent norm $\|u\|_{\mu}$, where

$$
\|u\|_{\mu}^{p}=\int_{\Omega}\left(|\nabla u|^{p}-\mu \frac{|u|^{p}}{|x|^{p}}\right) \mathrm{d} x, \quad \mu \in(-\infty, \bar{\mu}) .
$$

Therefore, for $1<p<N$, and $\mu<\bar{\mu}$, we can define the best Sobolev constant:

$$
S_{\mu}(\Omega)=\inf _{u \in \mathcal{D}_{0}^{1, p}(\Omega) \backslash\{0\}} \frac{\int_{\Omega}\left(|\nabla u|^{p}-\mu \frac{|u|^{p}}{|x|^{p}}\right) d x}{\left(\int_{\Omega}|u|^{p *} d x\right)^{\frac{p}{p *}}} .
$$

It is well known that $S_{\mu}(\Omega)=S_{\mu}\left(\mathbb{R}^{N}\right)=S_{\mu}$. Note that $S_{\mu}=S_{0}$ when $\mu \leq 0$ [3].

Such kind of problem with critical exponents and nonnegative weight functions has been extensively studied by many authors. We refer, e.g., in bounded domains and for $p=2$ to [4-6] and for $p>1$ to [7-11], while in $\mathbb{R}^{N}$ and for $p=2$ to [12,13], and for $p$ $>1$ to [3,14-17], and the references therein.

In the present paper, our research is mainly related to (1.1) with $1<q<p<N$, the critical exponent and weight functions $f, g$ that change sign on $\Omega$. When $p=2,1<q$ $<2, \mu \in[0, \bar{\mu}), f, g$ are sign changing and $\Omega$ is bounded, [18] studied (1.1) and obtained that there exists $\Lambda>0$ such that (1.1) has at least two positive solutions for all $\lambda \in(0$, $\Lambda$ ). For the case $p \neq 2$, [19] studied (1.1) and obtained the multiplicity of positive solutions when $1<q<p<N, \mu=0, f, g$ are sign changing and $\Omega$ is bounded. However, little has been done for this type of problem (1.1). Recently, Wang et al. [11] have studied (1.1) in a bounded domain $\Omega$ under the assumptions $1<q<p<N, N>p^{2}$, $-\infty<\mu<\bar{\mu}$ and $f, g$ are nonnegative. They also proved that there existence of $\Lambda_{0}>0$ such that for $\lambda \in\left(0, \Lambda_{0}\right)$, (1.1) possesses at least two positive solutions. In this paper, we study (1.1) and extend the results of $[11,18,19]$ to the more general case $1<q<p$ $<N,-\infty<\mu<\bar{\mu}, f, g$ are sign changing and $\Omega$ is a smooth domain (not necessarily bounded) in $\mathbb{R}^{N}(N \geq 3)$. By extracting the Palais-Smale sequence in the Nehari manifold, the existence of at least two positive solutions of (1.1) is verified.

The following assumptions are used in this paper:

$(\mathcal{H}) \mu<\bar{\mu}, \lambda>0,1<q<p<N, N \geq 3$.

$\left(f_{1}\right) f \in C(\bar{\Omega}) \cap L^{q *}(\Omega)\left(q^{*}=\frac{p^{*}}{p^{*}-q}\right) f^{+}=\max \{f, 0\} \neq 0$ in $\Omega$.

$\left(f_{2}\right)$ There exist $\beta_{0}$ and $\rho_{0}>0$ such that $B\left(x_{0} ; 2 \rho_{0}\right) \subset \Omega$ and $f(x) \geq \beta_{0}$ for all $x \in B\left(x_{0}\right.$; $\left.2 \rho_{0}\right)$

$\left(g_{1}\right) g \in C(\bar{\Omega}) \cap L^{\infty}(\Omega)$ and $g^{+}=\max \{g, 0\} \neq 0$ in $\Omega$.

$\left(g_{2}\right)$ There exist $x_{0} \in \Omega$ and $\beta>0$ such that

$$
\begin{array}{r}
|g|_{\infty}=g\left(x_{0}\right)=\max _{x \in \bar{\Omega}} g(x), \quad g(x)>0, \forall x \in \Omega, \\
g(x)=g\left(x_{0}\right)+o\left(\left|x-x_{0}\right|^{\beta}\right) \quad \text { as } x \rightarrow 0
\end{array}
$$

where $|\cdot|_{\infty}$ denotes the $L^{\infty}(\Omega)$ norm. 
Set

$$
\Lambda_{1}=\Lambda_{1}(\mu)=\left(\frac{p-q}{(p *-q)\left|g^{+}\right|_{\infty}}\right)^{\frac{p-q}{p *-p}}\left(\frac{p *-p}{(p *-q)\left|f^{+}\right|_{q *}}\right) S_{\mu}^{\frac{N}{p^{2}}(p-q)+\frac{q}{p}} .
$$

The main results of this paper are concluded in the following theorems. When $\Omega$ is an unbounded domain, the conclusions are new to the best of our knowledge.

Theorem 1.1 Suppose $(\mathcal{H}),\left(f_{1}\right)$ and $\left(g_{1}\right)$ hold. Then, (1.1) has at least one positive solution for all $\lambda \in\left(0, \Lambda_{1}\right)$.

Theorem 1.2 Suppose $(\mathcal{H}),\left(f_{1}\right)-\left(g_{2}\right)$ hold, and $\gamma$ is the constant defined as in Lemma 2.2. If $0 \leq \mu<\bar{\mu}, x_{0}=0$ and $\beta \geq p \gamma$, then (1.1) has at least two positive solutions for all $\lambda \in\left(0, \frac{q}{p} \Lambda_{1}\right)$.

Theorem 1.3 Suppose $(\mathcal{H}),\left(f_{1}\right)-\left(g_{2}\right)$ hold. If $\mu<0, x_{0} \neq 0, \beta \geq \frac{N-p}{p-1}$ and $N \leq p^{2}$, then (1.1) has at least two positive solutions for all $\lambda \in\left(0, \frac{q}{p} \Lambda_{1}(0)\right)$.

Remark 1.4 As $\Omega$ is a bounded smooth domain and $p=2$, the results of Theorems 1.1, 1.2 are improvements of the main results of [18].

Remark 1.5 As $\Omega$ is a bounded smooth domain and $p \neq 2, \mu=0$, then the results of Theorems 1.1, 1.2 in this case are the same as the known results in [19].

Remark 1.6 In this remark, we consider that $\Omega$ is a bounded domain. In [11], Wang et al. considered (1.1) with $\mu<\bar{\mu}, \lambda>0$ and $1<q<p<p^{2}<N$. As $0 \leq \mu<\bar{\mu}$ and 1 $\mathrm{w}<q<p<N$, the results of Theorems 1.1, 1.2 are improvements of the main results of [11]. As $\mu<0$ and $1<q<p<N \leq p^{2}$, Theorem 1.3 is the complement to the results in [[11], Theorem 1.3].

This paper is organized as follows. Some preliminaries and properties of the Nehari manifold are established in Sections 2 and 3, and Theorems 1.1-1.3 are proved in Sections 4-6, respectively. Before ending this section, we explain some notations employed in this paper. In the following argument, we always employ $C$ and $C_{i}$ to denote various positive constants and omit $\mathrm{d} x$ in integral for convenience. $B\left(x_{0} ; R\right)$ is the ball centered at $x_{0} \in \mathbb{R}^{N}$ with the radius $R>0,\left(\mathcal{D}_{0}^{1, p}(\Omega)\right)^{-1}$ denotes the dual space of $\mathcal{D}_{0}^{1, p}(\Omega)$, the norm in $L^{p}(\Omega)$ is denoted by $|\cdot|_{p}$, the quantity $O\left(\varepsilon^{t}\right)$ denotes $\left|O\left(\varepsilon^{t}\right) / \varepsilon^{t}\right| \leq C$, o $o\left(\varepsilon^{t}\right)$ means $\left|o\left(\varepsilon^{t}\right) / \varepsilon^{t}\right| \rightarrow 0$ as $\varepsilon \rightarrow 0$ and $o(1)$ is a generic infinitesimal value. In particular, the quantity $O_{1}\left(\varepsilon^{t}\right)$ means that there exist $C_{1}, C_{2}>0$ such that $C_{1} \varepsilon^{t} \leq O_{1}\left(\varepsilon^{t}\right) \leq C_{2} \varepsilon^{t}$ as $\varepsilon$ is small enough.

\section{Preliminaries}

Throughout this paper, $\left(f_{1}\right)$ and $\left(g_{1}\right)$ will be assumed. In this section, we will establish several preliminary lemmas. To this end, we first recall a result on the extremal functions of $S_{\mu, s}$.

Lemma 2.1 [16]Assume that $1<p<N$ and $0 \leq \mu<\bar{\mu}$. Then, the limiting problem

$$
\left\{\begin{array}{l}
-\Delta_{p} u-\mu \frac{u^{p-1}}{|x|^{p}}=u^{p *-1}, \text { in } \mathbb{R}^{N} \backslash\{0\}, \\
u \in \mathcal{D}^{1, p}\left(\mathbb{R}^{N}\right), \quad u>0, \text { in } \mathbb{R}^{N} \backslash\{0\},
\end{array}\right.
$$


has positive radial ground states

$$
V_{p, \mu, \varepsilon}(x)=\varepsilon^{-\frac{N-p}{p}} U_{p, \mu}\left(\frac{x}{\varepsilon}\right)=\varepsilon^{-\frac{N-p}{p}} U_{p, \mu}\left(\frac{|x|}{\varepsilon}\right), \quad \text { for all } \varepsilon>0,
$$

that satisfy

$$
\int_{\mathbb{R}^{N}}\left(\left|\nabla V_{p, \mu, \varepsilon}(x)\right|^{p}-\mu \frac{\left|V_{p, \mu, \varepsilon}(x)\right|^{p}}{|x|^{p}}\right)=\int_{\mathbb{R}^{N}}\left|V_{p, \mu, \varepsilon}(x)\right|^{p *}=S_{\mu}^{\frac{N}{p}} .
$$

Furthermore, $U_{p, \mu}(|x|)=U_{p, \mu}(r)$ is decreasing and has the following properties:

$$
\begin{gathered}
U_{p, \mu}(1)=\left(\frac{N(\bar{\mu}-\mu)}{N-p}\right)^{\frac{1}{p *-p}}, \\
\lim _{r \rightarrow 0^{+}} r^{a(\mu)} U_{p, \mu}(r)=c_{1}>0, \quad \lim _{r \rightarrow 0^{+}} r^{a(\mu)+1}\left|U_{p, \mu}^{\prime}(r)\right|=c_{1} a(\mu) \geq 0, \\
\lim _{r \rightarrow+\infty} r^{b(\mu)} U_{p, \mu}(r)=c_{2}>0, \quad \lim _{r \rightarrow+\infty} r^{b(\mu)+1}\left|U_{p, \mu}^{\prime}(r)\right|=c_{2} b(\mu)>0, \\
c_{3} \leq U_{p, \mu}(r)\left(r^{\frac{a(\mu)}{\delta}}+r^{\frac{b(\mu)}{\delta}}\right)^{\delta} \leq c_{4}, \quad \delta:=\frac{N-p}{p},
\end{gathered}
$$

where $c_{i}(i=1,2,3,4)$ are positive constants depending on $N, \mu$ and $p$, and $a(\mu)$ and $b(\mu)$ are the zeros of the function $h(t)=(p-1) t^{p}-(N-p) t^{p-1}+\mu, t \geq 0$, satisfying $0 \leq a(\mu)<\frac{N-p}{p}<b(\mu) \leq \frac{N-p}{p-1}$.

Take $\rho>0$ small enough such that $B(0 ; \rho) \subset \Omega$, and define the function

$$
u_{\varepsilon}(x)=\eta(x) V_{p, \mu, \varepsilon}(x)=\varepsilon^{-\frac{N-p}{p}} \eta(x) U_{p, \mu}\left(\frac{|x|}{\varepsilon}\right),
$$

where $\eta \in C_{0}^{\infty}\left(B(0 ; \rho)\right.$ is a cutoff function such that $\eta(x) \equiv 1$ in $B\left(0, \frac{\rho}{2}\right)$.

Lemma $2.2[9,20]$ Suppose $1<p<N$ and $0 \leq \mu<\bar{\mu}$. Then, the following estimates hold when $\varepsilon \rightarrow 0$.

$$
\begin{aligned}
\left\|u_{\varepsilon}\right\|_{\mu}^{p} & =S_{\mu}^{\frac{N}{p}}+O\left(\varepsilon^{p \gamma}\right), \\
\int_{\Omega}\left|u_{\varepsilon}\right|^{p *} & =S_{\mu}^{\frac{N}{p}}+O\left(\varepsilon^{p * \gamma}\right), \\
\int_{\Omega}\left|u_{\varepsilon}\right|^{q} & =\left\{\begin{array}{lr}
O_{1}\left(\varepsilon^{\theta}\right), & \frac{N}{b(\mu)}<q<p *, \\
O_{1}\left(\varepsilon^{\theta} \mid\right) \ln \varepsilon \mid, & q=\frac{N}{b(\mu)}, \\
O_{1}\left(\varepsilon^{q \gamma}\right), & 1 \leq q<\frac{N}{b(\mu)},
\end{array}\right.
\end{aligned}
$$

where $\delta=\frac{N-p}{p}, \theta=N-\frac{N-p}{p}$ qand $\gamma=b(\mu)-\delta$.

We also recall the following known result by Ben-Naoum, Troestler and Willem, which will be employed for the energy functional.

Lemma 2.3 [21] Let $\Omega$ be an domain, not necessarily bounded, in $\mathbb{R}^{N}, 1 \leq p<N$, $k(x) \in L^{\frac{p *}{p *-q}}(\Omega)^{a n d} k(x) \in L^{\frac{p *}{p *-q}}(\Omega)^{\text {Then, the functional }}$

$$
\mathcal{D}_{0}^{1, p}(\Omega) \rightarrow \mathbb{R}: u \mapsto \int_{\mathbb{R}^{N}} k(x)|u|^{q} \mathrm{~d} x
$$

is well-defined and weakly continuous. 


\section{Nehari manifold}

As $J_{\lambda}$ is not bounded below on $\mathcal{D}_{0}^{1, p}(\Omega)$, we need to study $J_{\lambda}$ on the Nehari manifold

$$
\mathcal{N}_{\lambda}=\left\{u \in \mathcal{D}_{0}^{1, p}(\Omega) \backslash\{0\}:\left\langle J_{\lambda}^{\prime}(u), u\right\rangle=0\right\} .
$$

Note that $\mathcal{N}_{\lambda}$ contains all solutions of (1.1) and $u \in \mathcal{N}_{\lambda}$ if and only if

$$
\|u\|_{\mu}^{p}-\lambda \int_{\Omega} f|u|^{q}-\int_{\Omega} g|u|^{p *}=0 .
$$

Lemma 3.1 $J_{\lambda}$ is coercive and bounded below on $\mathcal{N}_{\lambda}$.

Proof Suppose $u \in \mathcal{N}_{\lambda}$. From $\left(f_{1}\right)$, (3.1), the Hölder inequality and Sobolev embedding theorem, we can deduce that

$$
\begin{aligned}
J_{\lambda}(u) & =\frac{p *-p}{p p *} \|\left. u\right|_{\mu} ^{p}-\lambda \frac{p *-q}{p * q} \int_{\Omega} f|u|^{q} \\
& \geq \frac{1}{N}\|u\|_{\mu}^{p}-\lambda \frac{p *-q}{p * q}\left|f^{+}\right|_{q *}|u|_{p *}^{q} \\
& \geq \frac{1}{N}\|u\|_{\mu}^{p}-\lambda \frac{p *-q}{p * q}\left|f^{+}\right|_{q *} S_{\mu}^{-\frac{q}{p}}\|u\|_{\mu}^{q} .
\end{aligned}
$$

Thus, $J_{\lambda}$ is coercive and bounded below on $\mathcal{N}_{\lambda}$. $\square$

Define $\psi_{\lambda}(u)=\left\langle J_{\lambda}^{\prime}(u), u\right\rangle$. Then, for $u \in \mathcal{N}_{\lambda}$,

$$
\begin{aligned}
\left\langle\psi_{\lambda}^{\prime}(u), u\right\rangle & =p\|u\|_{\mu}^{p}-q \lambda \int_{\Omega} f|u|^{q}-p * \int_{\Omega} g|u|^{p *} \\
& =(p-q)\|u\|_{\mu}^{p}-(p *-q) \int_{\Omega} g|u|^{p *} \\
& =\lambda\left(p^{*}-q\right) \int_{\Omega} f|u|^{q}-(p *-p)\|u\|_{\mu}^{p} .
\end{aligned}
$$

Arguing as in [22], we split $\mathcal{N}_{\lambda}$ into three parts:

$$
\begin{aligned}
& \mathcal{N}_{\lambda}^{+}=\left\{u \in \mathcal{N}_{\lambda}:\left\langle\psi^{\prime}{ }_{\lambda}(u), u\right\rangle>0\right\}, \\
& \mathcal{N}_{\lambda}^{0}=\left\{u \in \mathcal{N}_{\lambda}:\left\langle\psi^{\prime}{ }_{\lambda}(u), u\right\rangle=0\right\}, \\
& \mathcal{N}_{\lambda}^{-}=\left\{u \in \mathcal{N}_{\lambda}:\left\langle\psi^{\prime}{ }_{\lambda}(u), u\right\rangle<0\right\} .
\end{aligned}
$$

Lemma 3.2 Suppose $u_{\lambda}$ is a local minimizer of $J_{\lambda}$ on $\mathcal{N}_{\lambda}$ and $u_{\lambda} \notin \mathcal{N}_{\lambda}^{0}$.

Then, $J_{\lambda}^{\prime}\left(u_{\lambda}\right)=\operatorname{Oin}\left(\mathcal{D}_{0}^{1, p}(\Omega)\right)^{-1}$.

Proof The proof is similar to [[23], Theorem 2.3] and is omitted.

Lemma $3.3 \mathcal{N}_{\lambda}^{0} \neq \emptyset$ ffor all $\lambda \in\left(0, \Lambda_{1}\right)$.

Proof We argue by contradiction. Suppose that there exists $\lambda \in\left(0, \Lambda_{1}\right)$ such that $\mathcal{N}_{\lambda}^{0} \neq \emptyset$. Then, the fact $u \in \mathcal{N}_{\lambda}^{0}$ and (3.3) imply that

$$
\|u\|_{\mu}^{p}=\frac{p *-q}{p-q} \int_{\Omega} g|u|^{p *},
$$

and

$$
\|u\|_{\mu}^{p}=\lambda \frac{p^{*}-q}{p^{*}-p} \int_{\Omega} f|u|^{q} .
$$


By $\left(f_{1}\right),\left(g_{1}\right)$, the Hölder inequality and Sobolev embedding theorem, we have that

$$
\|u\|_{\mu} \geq\left[\frac{p-q}{\left(p^{*}-q\right)\left|g^{+}\right|_{\infty}}\right]^{\frac{1}{p^{*}-p}} S_{\mu}^{\frac{N}{p^{2}}}
$$

and

$$
\|u\|_{\mu} \leq\left[\lambda \frac{p^{*}-q}{p^{*}-p}\left|f^{+}\right|_{q^{*}} S_{\mu}-\frac{q}{p}\right]^{\frac{1}{p-q}}
$$

Consequently,

$$
\lambda \geq\left(\frac{p-q}{\left(p^{*}-q\right)\left|g^{+}\right|_{\infty}}\right)^{\frac{p-q}{p^{*}-p}}\left(\frac{p^{*}-p}{\left(p^{*}-q\right)\left|f^{+}\right|_{q^{*}}}\right) S_{\mu}^{\frac{N}{p^{2}}(p-q)+\frac{q}{p}}=\Lambda_{1},
$$

which is a contradiction.

For each $u \in \mathcal{D}_{0}^{1, p}(\Omega)$ with $\int_{\Omega} g|u|^{p^{*}}>0$, we set

$$
t_{\max }=\left(\frac{(p-q)\|u\|_{\mu}^{p}}{\left(p^{*}-q\right) \int_{\Omega} g|u|^{p^{*}}}\right)^{\frac{1}{p^{*}-p}}>0
$$

Lemma 3.4 Suppose that $\lambda \in\left(0, \Lambda_{1}\right)$ and $u \in \mathcal{D}_{0}^{1, p}(\Omega)$ is a function satisfying with $\int_{\Omega} g|u|^{p^{*}}>0$.

(i) If $\int_{\Omega} f|u|^{q} \leq 0$, then there exists a unique $t^{-}>t_{\max }$ such that $t^{-} u \in \mathcal{N}_{\lambda}^{-}$and

$$
J_{\lambda}\left(t^{-} u\right)=\sup _{t \geq 0} J_{\lambda}(t u) .
$$

(ii) If $\int_{\Omega} f|u|^{q} \leq 0$, then there exists a unique $t^{ \pm}$such that $0<t^{+}<t_{\max }<t^{-}$, $t^{-} u \in \mathcal{N}_{\lambda}^{-}$and $t^{-} u \in \mathcal{N}_{\lambda}^{-}$. Moreover,

$$
J_{\lambda}\left(t^{+} u\right)=\inf _{0 \leq t \leq t_{\max }} J_{\lambda}(t u), \quad J_{\lambda}\left(t^{-} u\right)=\sup _{t \geq t^{+}} J_{\lambda}(t u) .
$$

Proof See Brown-Wu [[24], Lemma 2.6].

We remark that it follows Lemma 3.3, $\mathcal{N}_{\lambda}=\mathcal{N}_{\lambda}^{+} \cup \mathcal{N}_{\lambda}^{-}$for all $\lambda \in\left(0, \Lambda_{1}\right)$. Furthermore, by Lemma 3.4, it follows that $\mathcal{N}_{\lambda}^{+}$and $\mathcal{N}_{\lambda}^{-}$are nonempty, and by Lemma 3.1, we may define

$$
\alpha_{\lambda}=\inf _{u \in \mathcal{N}_{\lambda}} J_{\lambda}(u), \quad \alpha_{\lambda}^{+}=\inf _{u \in \mathcal{N}_{\lambda}^{+}} J_{\lambda}(u), \quad \alpha_{\lambda}^{-}=\inf _{u \in \mathcal{N}_{\lambda}^{-}} J_{\lambda}(u)
$$

Lemma 3.5 (i) If $\lambda \in\left(0, \Lambda_{1}\right)$, then we have $\alpha_{\lambda} \leq \alpha_{\lambda}^{+}<0$.

(ii) If $\lambda \in\left(0, \frac{q}{p} \Lambda_{1}\right)$, then $\alpha_{\lambda}^{-}>d_{0}$ for some positive constant $d_{0}$.

In particular, for each $\lambda \in\left(0, \frac{q}{p} \Lambda_{1}\right)$, we have $\alpha_{\lambda}=\alpha_{\lambda}^{+}<0<\alpha_{\lambda}^{-}$.

Proof (i) Suppose that $u \in \mathcal{N}_{\lambda}^{+}$. From (3.3), it follows that

$$
\frac{p-q}{p^{*}-q}\|u\|_{\mu}^{p}>\int_{\Omega} g|u|^{p^{*}}
$$


According to (3.1) and (3.4), we have

$$
\begin{aligned}
J_{\lambda}(u) & =\left(\frac{1}{p}-\frac{1}{q}\right)\|u\|_{\mu}^{p}+\left(\frac{1}{q}-\frac{1}{p^{*}}\right) \int_{\Omega} g|u|^{p^{*}} \\
& <\left[\left(\frac{1}{p}-\frac{1}{q}\right)+\left(\frac{1}{q}-\frac{1}{p^{*}}\right)\left(\frac{p-q}{p^{*}-q}\right)\right]\|u\|_{\mu}^{p} \\
& =-\frac{p-q}{q N}\|u\|_{\mu}^{p}<0 .
\end{aligned}
$$

By the definitions of $\alpha_{\lambda}$ and $\alpha_{\lambda}^{+}$, we get that $\alpha_{\lambda} \leq \alpha_{\lambda}^{+}<0$.

(ii) Suppose $\lambda \in\left(0, \frac{q}{p} \Lambda_{1}\right)$ and $u \in \mathcal{N}_{\lambda}^{-}$. Then, (3.3) implies that

$$
\frac{p-q}{p^{*}-q}\|u\|_{\mu}^{p}<\int_{\Omega}|u|^{p^{*}}
$$

Moreover, by $\left(g_{1}\right)$ and the Sobolev embedding theorem, we have

$$
\int_{\Omega} g|u|^{p^{*}} \leq\left|g^{+}\right|_{\infty} S_{\mu}^{-\frac{p^{*}}{p}}\|u\|_{\mu}^{p^{*}}
$$

From (3.5) and (3.6), it follows that

$$
\|u\|_{\mu}>\left(\frac{p-q}{\left(p^{*}-q\right)\left|g^{+}\right|_{\infty}}\right)^{\frac{1}{p^{*}-p}} S_{\mu}^{\frac{N}{p^{2}}} \text { for all } u \in \mathcal{N}_{\lambda}^{-} .
$$

By (3.2) and (3.7), we get

$$
\begin{aligned}
J_{\lambda}(u) \geq & \|\left. u\right|_{\mu} ^{q}\left[\frac{1}{N} \|\left. u\right|_{\mu} ^{p-q}-\lambda \frac{p^{*}-q}{p^{*} q}\left|f^{+}\right| q^{*} S_{\mu}^{-\frac{q}{p}}\right] \\
> & \left(\frac{p-q}{\left(p^{*}-q\right)\left|g^{+}\right|_{\infty}}\right)^{\frac{q}{p^{*}-p}} S_{\mu}^{\frac{q N}{p^{2}}}\left[\frac{1}{N}\left(\frac{p-q}{\left(p^{*}-q\right)\left|g^{+}\right|_{\infty}}\right)^{\frac{p-q}{p^{*}-p}} S_{\mu}^{\frac{N(p-q)}{p^{2}}}\right. \\
& \left.-\lambda \frac{p^{*}-q}{p^{*} q}\left|f^{+}\right|_{q^{*}} S_{\mu}^{-\frac{q}{p}}\right]
\end{aligned}
$$

which implies that

$$
J_{\lambda}(u)>d_{0} \text { for all } u \in \mathcal{N}_{\lambda}^{-},
$$

for some positive constant $d_{0}$. $\square$

Remark 3.6 If $\lambda \in\left(0, \frac{q}{p} \Lambda_{0}\right)$, then by Lemmas 3.4 and 3.5, for each $u \in \mathcal{D}_{0}^{1, p}(\Omega)$ with $\int_{\Omega} g|u|^{p^{*}}>0$, we can easily deduce that

$$
t^{-} u \in \mathcal{N}_{\lambda}^{-} \text {and } J_{\lambda}\left(t^{-} u\right)=\sup _{t \geq 0} J_{\lambda}(t u) \geq \alpha_{\lambda}^{-}>0 .
$$

\section{Proof of Theorem 1.1}

First, we define the Palais-Smale (simply by $(P S)$ ) sequences, $(P S)$-values and (PS)-conditions in $\mathcal{D}_{0}^{1, \phi}(\Omega)$ for $J_{\lambda}$ as follows:

Definition 4.1 (i) For $c \in \mathbb{R}$, a sequence $\left\{u_{n}\right\}$ is a $(P S)_{c}$-sequence in $\mathcal{D}_{0}^{1, p}(\Omega)$ for $J_{\lambda}$ if $J_{\lambda}$ $\left(u_{n}\right)=c+o(1)$ and $\left(J_{\lambda}\right)^{\prime}\left(u_{n}\right)=o(1)$ strongly in $\left(\mathcal{D}_{0}^{1, p}(\Omega)\right)^{-1}$ as $n \rightarrow \infty$. 
(ii) $c \in \mathbb{R}$ is a (PS)-value in $\mathcal{D}_{0}^{1, p}(\Omega)$ for $J_{\lambda}$ if there exists a $(P S)_{c}$-sequence in $\mathcal{D}_{0}^{1, p}(\Omega)$ for $J_{\lambda}$

(iii) $J_{\lambda}$ satisfies the $(P S)_{c}$-condition in $\mathcal{D}_{0}^{1, p}(\Omega)$ if any $(P S)_{c}$-sequence $\left\{u_{n}\right\}$ in $\mathcal{D}_{0}^{1, p}(\Omega)$ for $J_{\lambda}$ contains a convergent subsequence.

Lemma 4.2 (i) If $\lambda \in\left(0, \Lambda_{1}\right)$, then $J_{\lambda}$ has a (PS) $\alpha_{\alpha}$-sequence $\left\{u_{n}\right\} \subset \mathcal{N}_{\lambda}$.

(ii) If $\lambda \in\left(0, \frac{q}{p} \Lambda_{1}\right)$, then $J_{\lambda}$ has a $(P S)_{\alpha_{\lambda}}$-sequence $\left\{u_{n}\right\} \subset \mathcal{N}_{\lambda}^{-}$.

Proof The proof is similar to $[19,25]$ and the details are omitted. $\square$

Now, we establish the existence of a local minimum for $J_{\lambda}$ on $\mathcal{N}_{\lambda}$.

Theorem 4.3 Suppose that $N \geq 3, \mu<\bar{\mu}, 1<q<p<N$ and the conditions $\left(f_{1}\right),\left(g_{1}\right)$ hold. If $\lambda \in\left(0, \Lambda_{1}\right)$, then there exists $u_{\lambda} \in \mathcal{N}_{\lambda}^{+}$such that

(i) $J_{\lambda}\left(u_{\lambda}\right)=\alpha_{\lambda}=\alpha_{\lambda}^{+}$,

(ii) $u_{\lambda}$ is a positive solution of (1.1),

(iii) $\left\|u_{\lambda}\right\|_{\mu} \rightarrow 0$ as $\lambda \rightarrow 0^{+}$.

Proof By Lemma $4.2(i)$, there exists a minimizing sequence $\left\{u_{n}\right\} \subset \mathcal{N}_{\lambda}$ such that

$$
J_{\lambda}\left(u_{n}\right)=\alpha_{\lambda}+o(1) \quad \text { and } \quad J_{\lambda}^{\prime}\left(u_{n}\right)=o(1) \quad \text { in }\left(\mathcal{D}_{0}^{1, p}(\Omega)\right)^{-1}
$$

Since $J_{\lambda}$ is coercive on $\mathcal{N}_{\lambda}$ (see Lemma 2.1), we get that $\left(u_{n}\right)$ is bounded in $\mathcal{D}_{0}^{1, p}(\Omega)$. Passing to a subsequence, there exists $u_{\lambda} \in \mathcal{D}_{0}^{1, p}(\Omega)$ such that as $n \rightarrow \infty$

$$
\left\{\begin{array}{l}
u_{n} \rightarrow u_{\lambda} \text { weakly in } \mathcal{D}_{0}^{1, p}(\Omega), \\
u_{n} \rightarrow u_{\lambda} \text { weakly in } L^{p^{*}}(\Omega), \\
u_{n} \rightarrow u_{\lambda} \text { strongly in } L_{l o c}^{r}(\Omega) \text { for all } 1 \leq r<p^{*}, \\
u_{n} \rightarrow u_{\lambda} \text { a.e. in } \Omega .
\end{array}\right.
$$

By $\left(f_{1}\right)$ and Lemma 2.3, we obtain

$$
\lambda \int_{\Omega} f\left|u_{n}\right|^{q}=\lambda \int_{\Omega} f\left|u_{\lambda}\right|^{q}+o(1) \text { as } n \rightarrow \infty
$$

From (4.1)-(4.3), a standard argument shows that $u_{\lambda}$ is a critical point of $J_{\lambda}$. Furthermore, the fact $\left\{u_{n}\right\} \subset \mathcal{N}_{\lambda}$ implies that

$$
\lambda \int_{\Omega} f\left|u_{n}\right|^{q}=\frac{q\left(p^{*}-p\right)}{p\left(p^{*}-q\right)}\left\|u_{n}\right\|_{\mu}^{p}-\frac{p^{*} q}{p^{*}-q} J_{\lambda}\left(u_{n}\right) .
$$

Taking $n \rightarrow \infty$ in (4.4), by (4.1), (4.3) and the fact $\alpha_{\lambda}<0$, we get

$$
\lambda \int_{\Omega} f\left|u_{\lambda}\right|^{q} \geq-\frac{p^{*} q}{p^{*}-q} \alpha_{\lambda}>0 .
$$

Thus, $u_{\lambda} \in \mathcal{N}_{\lambda}$ is a nontrivial solution of (1.1).

Next, we prove that $u_{n} \rightarrow u_{\lambda}$ strongly in $\mathcal{D}_{0}^{1, p}(\Omega)$ and $J_{\lambda}\left(u_{\lambda}\right)=\alpha_{\lambda}$. From (4.3), the fact $u_{n}, u_{\lambda} \in \mathcal{N}_{\lambda}$ and the Fatou's lemma it follows that

$$
\begin{aligned}
\alpha_{\lambda} & \leq J_{\lambda}\left(u_{\lambda}\right)=\frac{1}{N}\left\|u_{\lambda}\right\|_{\mu}^{p}-\lambda \frac{p^{*}-q}{p^{*} q} \int_{\Omega} f\left|u_{\lambda}\right|^{q} \\
& \leq \liminf _{n \rightarrow \infty}\left(\frac{1}{N}\left\|u_{n}\right\|_{\mu}^{p}-\lambda \frac{p^{*}-q}{p^{*} q} \int_{\Omega} f\left|u_{n}\right|^{q}\right) \\
& =\liminf _{n \rightarrow \infty} J_{\lambda}\left(u_{n}\right)=\alpha_{\lambda},
\end{aligned}
$$


which implies that $J_{\lambda}\left(u_{\lambda}\right)=\alpha_{\lambda}$ and $\lim _{n \rightarrow \infty}\left\|u_{n}\right\|_{\mu}^{p}=\left\|u_{\lambda}\right\|_{\mu}^{p}$. Standard argument shows that $u_{n} \rightarrow u_{\lambda}$ strongly in $\mathcal{D}_{0}^{1, p}(\Omega)$. Moreover, $u_{\lambda} \in \mathcal{N}_{\lambda}^{+}$. Otherwise, if $u_{\lambda} \in \mathcal{N}_{\lambda}^{-}$, by Lemma 3.4, there exist unique $t_{\lambda}^{+}$and $t_{\lambda}^{-}$such that $t_{\lambda}^{+} u_{\lambda} \in \mathcal{N}_{\lambda}^{+}, t_{\lambda}^{-} u_{\lambda} \in \mathcal{N}_{\lambda}^{-}$and $t_{\lambda}^{+}<t_{\lambda}^{-}=1$. Since

$$
\frac{d}{d t} J_{\lambda}\left(t_{\lambda}^{+} u_{\lambda}\right)=0 \quad \text { and } \quad \frac{d^{2}}{d t^{2}} J_{\lambda}\left(t_{\lambda}^{+} u_{\lambda}\right)>0,
$$

there exists $\bar{t} \in\left(t_{\lambda}^{+}, t_{\lambda}^{-}\right)$such that $J_{\lambda}\left(t_{\lambda}^{+} u_{\lambda}\right)<J_{\lambda}\left(\bar{t} u_{\lambda}\right)$. By Lemma 3.4, we get that

$$
J_{\lambda}\left(t_{\lambda}^{+} u_{\lambda}\right)<J_{\lambda}\left(\bar{t} u_{\lambda}\right) \leq J_{\lambda}\left(t_{\lambda}^{-} u_{\lambda}\right)=J_{\lambda}\left(u_{\lambda}\right)
$$

which is a contradiction. If $u \in \mathcal{N}_{\lambda}^{+}$, then $|u| \in \mathcal{N}_{\lambda}^{+}$, and by $J_{\lambda}\left(u_{\lambda}\right)=J_{\lambda}\left(\left|u_{\lambda}\right|\right)=\alpha_{\lambda}$, we get $\left|u_{\lambda}\right| \in \mathcal{N}_{\lambda}^{+}$is a local minimum of $J_{\lambda}$ on $\mathcal{N}_{\lambda}$. Then, by Lemma 3.2, we may assume that $u_{\lambda}$ is a nontrivial nonnegative solution of (1.1). By Harnack inequality due to Trudinger [26], we obtain that $u_{\lambda}>0$ in $\Omega$. Finally, by (3.3), the Hölder inequality and Sobolev embedding theorem, we obtain

$$
\left\|u_{\lambda}\right\|_{\mu}^{p-q}<\lambda \frac{p^{*}-q}{p^{*}-p}\left|f^{+}\right|_{q^{*}} S_{\mu}^{-\frac{q}{p}} .
$$

which implies that $\left\|u_{\lambda}\right\|_{\mu} \rightarrow 0$ as $\lambda \rightarrow 0^{+}$. .

Proof of Theorem 1.1 From Theorem 4.3, it follows that the problem (1.1) has a positive solution $u_{\lambda} \in \mathcal{N}_{\lambda}^{+}$for all $\lambda \in\left(0, \Lambda_{0}\right)$. $\square$

\section{Proof of Theorem 1.2}

For $1<p<N$ and $\mu<\bar{\mu}$, let

$$
c^{*}=\frac{1}{N}\left|g^{+}\right|_{\infty}^{-\frac{N-p}{p}} S_{\mu}^{\frac{N}{p}} .
$$

Lemma 5.1 Suppose $\left\{u_{n}\right\}$ is a bounded sequence in $\mathcal{D}_{0}^{1, p}(\Omega)$. If $\left\{u_{n}\right\}$ is a $(P S)_{c}$-sequence for $J_{\lambda}$ with $c \in\left(0, c^{\prime \prime}\right)$, then there exists a subsequence of $\left\{u_{n}\right\}$ converging weakly to a nonzero solution of (1.1).

Proof Let $\left\{u_{n}\right\} \subset \mathcal{D}_{0}^{1, p}(\Omega)$ be a $(P S)_{c}$-sequence for $J_{\lambda}$ with $c \in\left(0, c^{\prime \prime}\right)$. Since $\left\{u_{n}\right\}$ is bounded in $\mathcal{D}_{0}^{1, p}(\Omega)$, passing to a subsequence if necessary, we may assume that as $n$ $\rightarrow \infty$

$$
\left\{\begin{array}{l}
u_{n} \rightarrow u_{0} \text { weakly in } \mathcal{D}_{0}^{1, p}(\Omega), \\
u_{n} \rightarrow u_{0} \text { weakly in } L^{p^{*}}(\Omega), \\
u_{n} \rightarrow u_{0} \text { strongly in } L_{l o c}^{r}(\Omega) \text { for } 1 \leq r<p^{*}, \\
u_{n} \rightarrow u_{0} \text { a.e. in } \Omega .
\end{array}\right.
$$

By $\left(f_{1}\right),\left(g_{1}\right),(5.1)$ and Lemma 2.3, we have that $J_{\lambda}^{\prime}\left(u_{0}\right)=0$ and

$$
\lambda \int_{\Omega} f\left|u_{n}\right|^{q}=\lambda \int_{\Omega} f\left|u_{0}\right|^{q}+o(1) \text { as } n \rightarrow \infty .
$$

Next, we verify that $u_{0} \neq 0$. Arguing by contradiction, we assume $u_{0} \equiv 0$. Since $J_{\lambda}^{\prime}\left(u_{n}\right)=o(1)$ as $n \rightarrow \infty$ and $\left\{u_{n}\right\}$ is bounded in $\mathcal{D}_{0}^{1, p}(\Omega)$, then by (5.2), we can deduce that 


$$
0=\left\langle\lim _{n \rightarrow \infty} J_{\lambda}^{\prime}\left(u_{n}\right), u_{n}\right\rangle=\lim _{n \rightarrow \infty}\left(\left\|u_{n}\right\|_{\mu}^{p}-\int_{\Omega} g\left|u_{n}\right|^{p^{*}}\right) .
$$

Then, we can set

$$
\lim _{n \rightarrow \infty}|| u_{n} \|_{\mu}^{p}=\lim _{n \rightarrow \infty} \int_{\Omega} g\left|u_{n}\right|^{p^{*}}=l
$$

If $l=0$, then we get $c=\lim _{n \rightarrow \infty} J_{\lambda}\left(u_{n}\right)=0$, which is a contradiction. Thus, we conclude that $l>0$. Furthermore, the Sobolev embedding theorem implies that

$$
\begin{aligned}
\left\|u_{n}\right\|_{\mu}^{p} & \geq S_{\mu}\left(\int_{\Omega} g\left|u_{n}\right|^{p^{*}}\right)^{\frac{p}{p^{*}}} \\
& \geq S_{\mu}\left(\int_{\Omega} \frac{g}{\left|g^{+}\right|_{\infty}}\left|u_{n}\right|^{p^{*}}\right)^{\frac{p}{p^{*}}} \\
& =S_{\mu}\left|g^{+}\right|_{\infty}^{-\frac{N-p}{N}}\left(\int_{\Omega} g\left|u_{n}\right|^{p^{*}}\right)^{\frac{p}{p^{*}}} .
\end{aligned}
$$

Then, as $n \rightarrow \infty$ we have $l=\lim _{n \rightarrow \infty}\left\|u_{n}\right\|_{\mu}^{p} \geq S_{\mu}\left|g^{+}\right|_{\infty}^{-\frac{N-p}{N}} \frac{p}{p^{*}}$, which implies that

$$
l \geq\left|g^{+}\right|_{\infty}^{-\frac{N-p}{p}} S_{\mu}^{\frac{N}{p}}
$$

Hence, from (5.2)-(5.4), we get

$$
\begin{aligned}
c & =\lim _{n \rightarrow \infty} J_{\lambda}\left(u_{n}\right) \\
& =\frac{1}{p} \lim _{n \rightarrow \infty}|| u_{n} \|_{\mu}^{p}-\frac{\lambda}{q} \lim _{n \rightarrow \infty} \int_{\Omega} f\left|u_{n}\right|^{q}-\frac{1}{p^{*}} \lim _{n \rightarrow \infty} \int_{\Omega} g\left|u_{n}\right|^{p^{*}} \\
& =\left(\frac{1}{p}-\frac{1}{p^{*}}\right) l \\
& \geq \frac{1}{N}\left|g^{+}\right|_{\infty}^{-\frac{N-p}{p}} S_{\mu}^{\frac{N}{p}} .
\end{aligned}
$$

This is contrary to $c<c$. Therefore, $u_{0}$ is a nontrivial solution of (1.1). $\square$

Lemma 5.2 Suppose $(\mathcal{H})$ and $\left(f_{1}\right)-\left(g_{2}\right)$ hold. If $0<\mu<\bar{\mu}, x_{0}=0$ and $\beta \geq p \gamma$, then for any $\lambda>0$, there exists $v_{\lambda} \in \mathcal{D}_{0}^{1, p}(\Omega)$ such that

$$
\sup _{t \geq 0} J_{\lambda}\left(t v_{\lambda}\right)<c^{*}
$$

In particular, $\alpha_{\lambda}^{-}<c^{*}$ for all $\lambda \in\left(0, \Lambda_{1}\right)$.

Proof From [[11], Lemma 5.3], we get that if $\varepsilon$ is small enough, there exist $t_{\varepsilon}>0$ and the positive constants $C_{i}(i=1,2)$ independent of $\varepsilon$, such that

$$
\sup _{t \geq 0} J_{\lambda}\left(t u_{\varepsilon}\right)=J_{\lambda}\left(t_{\varepsilon} u_{\varepsilon}\right) \text { and } 0<C_{1} \leq t_{\varepsilon} \leq C_{2}<\infty .
$$


By $\left(g_{2}\right)$, we conclude that

$$
\begin{aligned}
\left.\left|\int_{\Omega} g(x)\right| u_{\varepsilon}\right|^{p^{*}}-\int_{\Omega} g(0)\left|u_{\varepsilon}\right|^{p^{*}} \mid & \leq \int_{\Omega}|g(x)-g(0)|\left|u_{\varepsilon}\right|^{p^{*}} \\
& =O\left(\int_{B(0 ; \rho)}|x|^{\beta}\left|u_{\varepsilon}\right|^{p^{*}}\right) \\
& =O\left(\varepsilon^{\beta}\right),
\end{aligned}
$$

which together with Lemma 2.2 implies that

$$
\int_{\Omega} g(x)\left|u_{\varepsilon}\right|^{p^{*}}=g(0) S_{\mu}^{\frac{N}{p}}+O\left(\varepsilon^{p^{*} \gamma}\right)+O\left(\varepsilon^{\beta}\right) .
$$

From the fact $\lambda>0,1<q<p, \beta \geq p \gamma$ and

$$
\max _{t \geq 0}\left(\frac{t^{p}}{p} B_{1}-\frac{t^{p^{*}}}{p^{*}} B_{2}\right)=\frac{1}{N} B_{1}^{\frac{N}{p}} B_{2}^{-\frac{N-p}{p}}, \quad B_{1}>0, B_{2}>0,
$$

and by Lemma 2.2 , (5.7) and $\left(f_{2}\right)$, we get

$$
\begin{aligned}
J_{\lambda}\left(t_{\varepsilon} u_{\varepsilon}\right)= & \frac{t_{\varepsilon}^{p}}{p} \|\left. u_{\varepsilon}\right|_{\mu} ^{p}-\frac{t_{\varepsilon}^{p^{*}}}{p^{*}} \int_{\Omega} g\left|u_{\varepsilon}\right|^{p^{*}}-\lambda \frac{t_{\varepsilon}^{q}}{q} \int_{\Omega} f\left|u_{\varepsilon}\right|^{q} \\
\leq & \frac{1}{N}\left\|u_{\varepsilon}\right\|_{\mu}^{\frac{N}{p}}\left(\int_{\Omega} g \mid u_{\varepsilon} p^{p^{*}}\right)^{-\frac{N-p}{p}}-\lambda \frac{C_{1}^{q}}{q} \beta_{0} \int_{\Omega}\left|u_{\varepsilon}\right|^{q} \\
= & \frac{1}{N}\left(S_{\mu}^{\frac{N}{p}}+O\left(\varepsilon^{p \gamma}\right)\right)^{\frac{N}{p}}\left(g(0) S_{\mu}^{\frac{N}{p}}+O\left(\varepsilon^{p^{*} \gamma}\right)+O\left(\varepsilon^{\beta}\right)\right)^{-\frac{N-p}{p}} \\
& -\lambda \frac{C_{1}^{q}}{q} \beta_{0} \int_{\Omega}\left|u_{\varepsilon}\right|^{q} \\
= & \frac{1}{N} g(0)^{-\frac{N-p}{p}} S_{\mu}^{\frac{N}{p}}+O\left(\varepsilon^{p \gamma}\right)+O\left(\varepsilon^{\beta}\right)-\lambda \frac{C_{1}^{q}}{q} \beta_{0} \int_{\Omega}\left|u_{\varepsilon}\right|^{q} .
\end{aligned}
$$

By (5.6) and (5.8), we have that

$$
\sup _{t \geq 0} J_{\lambda}\left(t u_{\varepsilon}\right) \leq c^{*}+O\left(\varepsilon^{p \gamma}\right)+O\left(\varepsilon^{\beta}\right)-\lambda \frac{C_{1}^{q}}{q} \beta_{0} \int_{\Omega}\left|u_{\varepsilon}\right|^{q} .
$$

(i) If $1<q<\frac{N}{b(\mu)}$, then by Lemma 2.2 and $\gamma=b(\mu)-\delta=b(\mu)-\frac{N-p}{p}>0$ we have that

$$
\int_{\Omega}\left|u_{\varepsilon}\right|^{q}=O_{1}\left(\varepsilon^{q \gamma}\right)
$$

Combining this with (5.9), for any $\lambda>0$, we can choose $\varepsilon_{\lambda}$ small enough such that

$$
\sup _{t \geq 0} J_{\lambda}\left(t u_{\varepsilon_{\lambda}}\right)<c^{*} \text {. }
$$

(ii) If $\frac{N}{b(\mu)} \leq q<p$, then by Lemma 2.2 and $\gamma>0$ we have that

$$
\int_{\Omega}\left|u_{\varepsilon}\right|^{q}= \begin{cases}O_{1}\left(\varepsilon^{\theta}\right), & q>\frac{N}{b(\mu)}, \\ O_{1}\left(\varepsilon^{\theta}|\ln \varepsilon|\right), & q=\frac{N}{b(\mu)},\end{cases}
$$


and

$$
p \gamma=b(\mu) p+p-N>N+\left(1-\frac{N}{p}\right) q=\theta
$$

Combining this with (5.9), for any $\lambda>0$, we can choose $\varepsilon_{\lambda}$ small enough such that

$$
\sup _{t \geq 0} J_{\lambda}\left(t u_{\varepsilon_{\lambda}}\right)<c^{*}
$$

From (i) and (ii), (5.5) holds by taking $v_{\lambda}=u_{\varepsilon_{\lambda}}$.

In fact, by $\left(f_{2}\right),\left(g_{2}\right)$ and the definition of $u_{\varepsilon_{\lambda}}$, we have that

$$
\int_{\Omega} f\left|u_{\varepsilon_{\lambda}}\right|^{q}>0 \text { and } \int_{\Omega} g\left|u_{\varepsilon_{\lambda}}\right|^{p^{*}}>0 .
$$

From Lemma 3.4, the definition of $\alpha_{\lambda}^{-}$and (5.5), for any $\lambda \in\left(0, \Lambda_{0}\right)$, there exists $t_{\varepsilon_{\lambda}}>0$ such that $t_{\varepsilon_{\lambda}} u_{\varepsilon_{\lambda}} \in \mathcal{N}_{\lambda}^{-}$and

$$
\alpha_{\lambda}^{-} \leq J_{\lambda}\left(t_{\varepsilon_{\lambda}} u_{\varepsilon_{\lambda}}\right) \leq \sup _{t \geq 0} J_{\lambda}\left(t t_{\varepsilon_{\lambda}} u_{\varepsilon_{\lambda}}\right)<c^{*} .
$$

The proof is thus complete. $\square$

Now, we establish the existence of a local minimum of $J_{\lambda}$ on $\mathcal{N}_{\lambda}^{-}$.

Theorem 5.3 Suppose $(\mathcal{H})$ and $\left(f_{1}\right)-\left(g_{2}\right)$ hold. If $0<\mu<\bar{\mu}, x_{0}=0, \beta \geq p \gamma$ and $\lambda \in\left(0, \frac{q}{p} \Lambda_{1}\right)$, then there exists $U_{\lambda} \in \mathcal{N}_{\lambda}^{-}$such that

(i) $J_{\lambda}\left(U_{\lambda}\right)=\alpha_{\lambda}^{-}$,

(ii) $U_{\lambda}$ is a positive solution of (1.1).

Proof If $\lambda \in\left(0, \frac{q}{p} \Lambda_{1}\right)$, then by Lemmas 3.5 (ii), 4.2 (ii) and 5.2 , there exists a $\left\{u_{n}\right\} \subset \mathcal{N}_{\lambda}^{-}$-sequence $\left\{u_{n}\right\} \subset \mathcal{N}_{\lambda}^{-}$in $\mathcal{D}_{0}^{1, p}(\Omega)$ for $J_{\lambda}$ with $\alpha_{\lambda}^{-} \in\left(0, c^{*}\right)$. Since $J_{\lambda}$ is coercive on $\mathcal{N}_{\lambda}^{-}$(see Lemma 3.1), we get that $\left\{u_{n}\right\}$ is bounded in $\mathcal{D}_{0}^{1, p}(\Omega)$. From Lemma 5.1, there exists a subsequence still denoted by $\left\{u_{n}\right\}$ and a nontrivial solution $U_{\lambda} \in \mathcal{D}_{0}^{1, p}(\Omega)$ of (1.1) such that $u_{n} \rightarrow U_{\lambda}$ weakly in $\mathcal{D}_{0}^{1, p}(\Omega)$.

First, we prove that $U_{\lambda} \in \mathcal{N}_{\lambda}^{-}$. On the contrary, if $U_{\lambda} \in \mathcal{N}_{\lambda}^{+}$, then by $\mathcal{N}_{\lambda}^{-} \cup\{0\}$ is closed in $\mathcal{D}_{0}^{1, p}(\Omega)$, we have $\left\|U_{\lambda}\right\|_{\mu}<\lim \inf _{n \rightarrow \infty}\left\|u_{n}\right\|_{\mu}$. From $\left(g_{2}\right)$ and $U_{\lambda} \neq 0$ in $\Omega$, we have $\int_{\Omega} g\left|U_{\lambda}\right|^{p^{*}}>0$. Thus, by Lemma 3.4, there exists a unique $t_{\lambda}$ such that $t_{\lambda} U_{\lambda} \in \mathcal{N}_{\lambda}^{-}$. If $u \in \mathcal{N}_{\lambda}$, then it is easy to see that

$$
J_{\lambda}(u)=\frac{1}{N}\|u\|_{\mu}^{p}-\lambda\left(\frac{p^{*}-q}{p^{*} q}\right) \int_{\Omega} f|u|^{q} .
$$

From Remark 3.6, $u_{n} \in \mathcal{N}_{\lambda}^{-}$and (5.10), we can deduce that

$$
\alpha_{\lambda}^{-} \leq J_{\lambda}\left(t_{\lambda} U_{\lambda}\right)<\lim _{n \rightarrow \infty} J_{\lambda}\left(t_{\lambda} u_{n}\right) \leq \lim _{n \rightarrow \infty} J_{\lambda}\left(u_{n}\right)=\alpha_{\lambda}^{-} .
$$

This is a contradiction. Thus, $U_{\lambda} \in \mathcal{N}_{\lambda}^{-}$.

Next, by the same argument as that in Theorem 4.3, we get that $u_{n} \rightarrow U_{\lambda}$ strongly in $\mathcal{D}_{0}^{1, p}(\Omega)$ and $J_{\lambda}\left(U_{\lambda}\right)=\alpha_{\lambda}^{-}>0$ for all $\lambda \in\left(0, \frac{q}{p} \Lambda_{1}\right)$. Since $J_{\lambda}\left(U_{\lambda}\right)=J_{\lambda}\left(\left|U_{\lambda}\right|\right)$ and $\left|U_{\lambda}\right| \in \mathcal{N}_{\lambda}^{-}$, by Lemma 3.2 , we may assume that $U_{\lambda}$ is a nontrivial nonnegative solution 
of (1.1). Finally, by Harnack inequality due to Trudinger [26], we obtain that $U_{\lambda}$ is a positive solution of (1.1). $\square$

Proof of Theorem 1.2 From Theorem 4.3, we get the first positive solution $u_{\lambda} \in \mathcal{N}_{\lambda}^{+}$ for all $\lambda \in\left(0, \Lambda_{0}\right)$. From Theorem 5.3, we get the second positive solution $U_{\lambda} \in \mathcal{N}_{\lambda}^{+}$ for all $\lambda \in\left(0, \frac{q}{p} \Lambda_{0}\right)$. Since $\mathcal{N}_{\lambda}^{-} \cap \mathcal{N}_{\lambda}^{-}=\emptyset$, this implies that $u_{\lambda}$ and $U_{\lambda}$ are distinct. $\square$

\section{Proof of Theorem 1.3}

In this section, we consider the case $\mu \leq 0$. In this case, it is well-known $S_{\mu}=S_{0}$ where $S_{\mu}$ is defined as in (1.2). Thus, we have $c^{*}=\frac{1}{N}\left|g^{+}\right|_{\infty}^{-\frac{N-p}{p}} S_{0}^{\frac{N}{p}}$ when $\mu \leq 0$.

Lemma 6.1 Suppose $(\mathcal{H})$ and $\left(f_{1}\right)-\left(g_{2}\right)$ hold. If $N \leq p^{2}, \mu<0, x_{0} \neq 0$ and $\frac{\beta}{p} \geq \tilde{\gamma}:=\frac{N-p}{p(p-1)}$, then for any $\lambda>0$ and $\mu<0$, there exists $v_{\lambda, \mu} \in \mathcal{D}_{0}^{1, p}(\Omega)$ such that

$$
\sup _{t \geq 0} J_{\lambda}\left(t v_{\lambda, \mu}\right)<c^{*} .
$$

In particular, $\alpha_{\lambda}^{-}<c^{*}$ for all $\lambda \in\left(0, \Lambda_{1}\right)$.

Proof Note that $\mathrm{S}_{0}$ has the following explicit extremals [27]:

$$
V_{\varepsilon}(x)=\bar{C} \varepsilon^{-\frac{N-p}{p}}\left(1+\left(\frac{\left|x-x_{0}\right|}{\varepsilon}\right)^{\frac{p}{p-1}}\right)^{-\frac{N-p}{p}}, \quad \forall \varepsilon>0, x_{0} \in \mathbb{R}^{N},
$$

where $\bar{C}>0$ is a particular constant. Take $\rho>0$ small enough such that $B\left(x_{0} ; \rho\right) \subset$ $\Omega \backslash\{0\}$ and set $\tilde{u}_{\varepsilon}(x)=\varphi(x) V_{\varepsilon}(x)$, where $\varphi(x) \in C_{0}^{\infty}\left(B\left(x_{0} ; \rho\right)\right.$ is a cutoff function such that $\phi(x) \equiv 1$ in $B\left(x_{0} ; \rho / 2\right)$. Arguing as in Lemma 2.2, we have

$$
\begin{aligned}
& \int_{\Omega}\left|\nabla \tilde{u}_{\varepsilon}\right|^{p}=S_{0}^{\frac{N}{p}}+O\left(\varepsilon^{p \tilde{\gamma}}\right), \\
& \int_{\Omega}\left|\tilde{u}_{\varepsilon}\right|^{p^{*}}=S_{0}^{\frac{N}{\bar{p}}}++O\left(\varepsilon^{\beta^{*} \tilde{\gamma}}\right), \\
& \int_{\Omega}\left|\tilde{u}_{\varepsilon}\right|^{q}=\left\{\begin{array}{lr}
O_{1}\left(\varepsilon^{\theta}\right), & \frac{N(p-1)}{N-p}<q<p^{*}, \\
O_{1}\left(\varepsilon^{\theta}|\ln \varepsilon|\right), & q=\frac{N(p-1)}{N-p}, \\
O_{1}\left(\varepsilon^{q \tilde{\gamma}}\right), & 1 \leq q<\frac{N(p-1)}{N-p},
\end{array}\right.
\end{aligned}
$$

where $\theta=N-\frac{N-p}{p} q$. Note that $\beta \geq p \tilde{\gamma}, p^{*} \tilde{\gamma}>p \tilde{\gamma}$. Arguing as in Lemma 5.2, we deduce that there exists $\tilde{t}_{\varepsilon}$ satisfying $0<C_{1} \leq \tilde{t}_{\varepsilon} \leq C_{2}$, such that

$$
\begin{aligned}
J_{\lambda}\left(t \tilde{u}_{\varepsilon}\right) \leq & \sup _{t \geq 0} J_{\lambda}\left(t \tilde{u}_{\varepsilon}\right)=J_{\lambda}\left(\tilde{t}_{\varepsilon} \tilde{u}_{\varepsilon}\right) \\
= & \frac{\tilde{t}_{\varepsilon}^{p}}{p} \int_{\Omega}\left|\nabla \tilde{u}_{\varepsilon}\right|^{p}-\left.\frac{\tilde{t}_{\varepsilon}^{*}}{p^{*}} \int_{\Omega} g\left|\tilde{u}_{\varepsilon} p^{p^{*}}-\lambda \frac{\tilde{t}_{\varepsilon}^{q}}{q} \int_{\Omega} f\right| \tilde{u}_{\varepsilon}\right|^{q}-\mu \frac{\tilde{t}_{\varepsilon}^{p}}{p} \int_{\Omega} \frac{\left|\tilde{u}_{\varepsilon}\right|^{p}}{|x|^{p}} \\
\leq & \frac{1}{N} g\left(x_{0}\right)^{-\frac{N-p}{p}} S_{\mu}^{\frac{N}{p}}+O\left(\varepsilon^{p \tilde{\gamma}}\right)-\lambda \frac{C_{1}^{q}}{q} \beta_{0} \int_{\Omega}\left|\tilde{u}_{\varepsilon}\right|^{q} \\
& -\mu|| x_{0}|-\rho|^{-p} \frac{C_{2}^{p}}{p} \int_{\Omega}\left|\tilde{u}_{\varepsilon}\right|^{p} .
\end{aligned}
$$


From $(\mathcal{H}), N \leq p^{2}$ and (6.4), we can deduce that

$$
1<q \tilde{\gamma}<p \tilde{\gamma}=\frac{N-p}{p-1} \leq p \leq \frac{N(p-1)}{N-p}
$$

and

$$
\int_{\Omega}\left|\tilde{u}_{\varepsilon}\right|^{q}=O_{1}\left(\varepsilon^{q \tilde{\gamma}}\right) \text { and } \int_{\Omega}\left|\tilde{u}_{\varepsilon}\right|^{p}=\left\{\begin{array}{lr}
O_{1}\left(\varepsilon^{p}|\ln \varepsilon|\right), & p=\frac{N(p-1)}{N-p} \\
O_{1}\left(\varepsilon^{p \tilde{\gamma}}\right), & 1<p<\frac{N(p-1)}{N-p}
\end{array}\right.
$$

Combining this with (6.5), for any $\lambda>0$ and $\mu<0$, we can choose $\varepsilon_{\lambda, \mu}$ small enough such that

$$
\sup _{t \geq 0} J_{\lambda}\left(t \tilde{u}_{\varepsilon_{\lambda, \mu}}\right)<\frac{1}{N} g\left(x_{0}\right)^{-\frac{N-p}{p}} S_{0}^{\frac{N}{p}}=c^{*} .
$$

Therefore, (6.1) holds by taking $v_{\lambda, \mu}=\tilde{u}_{\varepsilon_{\lambda, \mu}}$.

In fact, by $\left(f_{2}\right),\left(g_{2}\right)$ and the definition of $\tilde{u}_{\varepsilon_{\lambda, \mu}}$, we have that

$$
\int_{\Omega} f\left|\tilde{u}_{\varepsilon_{\lambda, \mu}}\right|^{q}>0 \text { and } \quad \int_{\Omega} g\left|\tilde{u}_{\varepsilon_{\lambda, \mu}}\right|^{p^{*}}>0 .
$$

From Lemma 3.4, the definition of $\alpha_{\lambda}^{-}$and (6.1), for any $\lambda \in\left(0, \Lambda_{0}\right)$ and $\mu<0$, there exists $t_{\varepsilon_{\lambda, \mu}}>0$ such that $t_{\varepsilon_{\lambda, \mu}} \tilde{u}_{\varepsilon_{\lambda, \mu}} \in \mathcal{N}_{\lambda}^{-}$and

$$
\alpha_{\lambda}^{-} \leq J_{\lambda}\left(t_{\varepsilon_{\lambda, \mu}} \tilde{u}_{\varepsilon_{\lambda, \mu}}\right) \leq \sup _{t \geq 0} J_{\lambda}\left(t t_{\varepsilon_{\lambda, \mu}} \tilde{u}_{\varepsilon_{\lambda, \mu}}\right)<c^{*} .
$$

The proof is thus complete.

Proof of Theorem 1.3 Let $\Lambda_{1}(0)$ be defined as in (1.3). Arguing as in Theorems 4.3 and 5.3, we can get the first positive solution $\tilde{u}_{\lambda} \in \mathcal{N}_{\lambda}^{+}$for all $\lambda \in\left(0, \Lambda_{1}(0)\right)$ and the second positive solution $\tilde{U}_{\lambda} \in \mathcal{N}_{\lambda}^{-}$for all $\lambda \in\left(0, \frac{q}{p} \Lambda_{1}(0)\right)$. Since $\mathcal{N}_{\lambda}^{+} \cap \mathcal{N}_{\lambda}^{-}=\emptyset$, this implies that $\tilde{u}_{\lambda}$ and $\tilde{U}_{\lambda}$ are distinct.

\section{Acknowledgements}

The author is grateful for the referee's valuable suggestions.

Received: 13 April 2011 Accepted: 19 October 2011 Published: 19 October 2011

\section{References}

1. Hardy, G, Littlewood, J, Polya, G: Inequalities, Reprint of the 1952 edition, Cambridge Math. Lib. Cambridge University Press, Cambridge (1988)

2. Caffarelli, L, Kohn, R, Nirenberg, L: First order interpolation inequality with weights. Compos Math. 53, $259-275$ (1984)

3. Abdellaoui, B, Felli, V, Peral, I: Existence and non-existence results for quasilinear elliptic equations involving the $p$ Laplacian. Boll Unione Mat Ital Scz B. 9, 445-484 (2006)

4. Cao, D, Han, P: Solutions for semilinear elliptic equations with critical exponents and Hardy potential. J Differ Equ. 205, 521-537 (2004). doi:10.1016/j.jde.2004.03.005

5. Ghoussoub, N, Robert, F: The effect of curvature on the best constant in the Hardy-Sobolev inequalities. Geom Funct Anal. 16, 897-908 (2006)

6. Kang, D, Peng, S: Solutions for semilinear elliptic problems with critical Sobolev-Hardy exponents and Hardy potential. Appl Math Lett. 18, 1094-1100 (2005). doi:10.1016/j.aml.2004.09.016

7. Cao, D, Kang, D: Solutions of quasilinear elliptic problems involving a Sobolev exponent and multiple Hardy-type terms. J Math Anal Appl. 333, 889-903 (2007). doi:10.1016/j.jmaa.2006.12.005

8. Ghoussoub, N, Yuan, C: Multiple solutions for quasi-linear PDEs involving the critical Sobolev and Hardy exponents. Trans Am Math Soc. 352, 5703-5743 (2000), doi:10.1090/S0002-9947-00-02560-5

9. Han, P: Quasilinear elliptic problems with critical exponents and Hardy terms. Nonlinear Anal. 61, 735-758 (2005). doi:10.1016/j.na.2005.01.030

10. Kang, D: On the quasilinear elliptic problems with critical Sobolev-Hardy exponents and Hardy terms. Nonlinear Anal. 68, 1973-1985 (2008). doi:10.1016/j.na.2007.01.024 
11. Wang, L, Wei, Q, Kang, D: Multiple positive solutions for p-Laplace elliptic equations involving concave-convex nonlinearities and a Hardytype term. Nonlinear Anal. 74, 626-638 (2011). doi:10.1016/j.na.2010.09.017

12. Felli, $V$, Terracini, S: Elliptic equations with multi-singular inverse-square potentials and critical nonlinearity. Commun Part Differ Equ. 31, 469-495 (2006). doi:10.1080/03605300500394439

13. Li, J: Existence of solution for a singular elliptic equation with critical Sobolev-Hardy exponents. Intern J Math Math Sci. 20, 3213-3223 (2005)

14. Filippucci, R, Pucci, P, Robert, F: On a p-Laplace equation with multiple critical nonlinearities. J Math Pures Appl. 91 156-177 (2009). doi:10.1016/j.matpur.2008.09.008

15. Gazzini, M, Musina, R: On a Sobolev-type inequality related to the weighted p-Laplace operator. J Math Anal Appl. 352, 99-111 (2009). doi:10.1016/j.jmaa.2008.06.021

16. Kang, D: Solution of the quasilinear elliptic problem with a critical Sobolev-Hardy exponent and a Hardy term. J Math Anal Appl. 341, 764-782 (2008). doi:10.1016/j.jmaa.2007.10.058

17. Pucci, $P$, Servadei, R: Existence, non-existence and regularity of radial ground states for p-Laplace weights. Ann Inst $H$ Poincaré Anal Non Linéaire. 25, 505-537 (2008). doi:10.1016/j.anihpc.2007.02.004

18. Hsu, TS, Lin, HL: Multiple positive solutions for singular elliptic equations with concave-convex nonlinearities and signchanging weights. Boundary Value Problems 2009, 17 (2009). Article ID 584203)

19. Hsu, TS: Multiplicity results for $p$-Laplacian with critical nonlinearity of concave-convex type and sign-changing weight functions. Abstr Appl Anal 2009, 24 (2009). Article ID 652109)

20. Kang, D, Huang, Y, Liu, S: Asymptotic estimates on the extremal functions of a quasilinear elliptic problem. J S Cent Univ Natl. 27, 91-95 (2008)

21. Ben-Naoum, AK, Troestler, C, Willem, M: Extrema problems with critical Sobolev exponents on unbounded domains. Nonlinear Anal. 26, 823-833 (1996). doi:10.1016/0362-546X(94)00324-B

22. Tarantello, G: On nonhomogeneous elliptic involving critical Sobolev exponent. Ann Inst H Poincaré Anal Non Linéaire. 9, 281-304 (1992)

23. Brown, KJ, Zhang, Y: The Nehari manifold for a semilinear elliptic equation with a sign-changing weigh function. J Differ Equ. 193, 481-499 (2003). doi:10.1016/50022-0396(03)00121-9

24. Brown, KJ, Wu, TF: A semilinear elliptic system involving nonlinear boundary condition and sign-changing weigh function. J Math Anal Appl. 337, 1326-1336 (2008). doi:10.1016/j.jmaa.2007.04.064

25. Wu, TF: On semilinear elliptic equations involving concave-convex nonlinearities and sign-changing weight function. J Math Anal Appl. 318, 253-270 (2006). doi:10.1016/j.jmaa.2005.05.057

26. Trudinger, NS: On Harnack type inequalities and their application to quasilinear elliptic equations. Commun Pure Appl Math. 20, 721-747 (1967). doi:10.1002/cpa.3160200406

27. Talenti, G: Best constant in Sobolev inequality. Ann Mat Pura Appl. 110, 353-372 (1976). doi:10.1007/BF02418013

doi:10.1186/1687-2770-2011-37

Cite this article as: Hsu: Multiple positive solutions for a class of quasi-linear elliptic equations involving concave-convex nonlinearities and Hardy terms. Boundary Value Problems 2011 2011:37.

\section{Submit your manuscript to a SpringerOpen ${ }^{\odot}$ journal and benefit from:}

- Convenient online submission

- Rigorous peer review

- Immediate publication on acceptance

- Open access: articles freely available online

- High visibility within the field

- Retaining the copyright to your article

Submit your next manuscript at $\gg$ springeropen.com 